\title{
Charge and Electrical Double Layer Formation in a Nonpolar Solvent Using a Nonionic Surfactant
}

\author{
Mahmoud Khademi, Sammi Sham Yin Cheng, and Dominik P. J. Barz* \\ Department of Chemical Engineering, Queen's University, Kingston, ON, K7L 3N6, \\ Canada
}

E-mail: dominik.barz@queensu.ca

\begin{abstract}
In this work, we study the charge formation and the characteristics of the electrical double layer in a nonpolar medium using electrical impedance spectroscopy. To stabilize the free ionic species, a nonionic surfactant is added to the system. The conductivity and permittivity of the medium are obtained from high- to medium-frequency impedance data. Based on the correlation between (viscosity-adjusted) conductivity and surfactant concentration, we conclude that charge formation occurs due to a disproportionation mechanism. We accordingly estimate the concentration of the charge carriers in the sample and the Debye length of the diffuse double layer. The capacitance of the electrical double layer can be extracted from the low-frequency impedance data. We use this data to calculate the electrode distance of an equivalent parallel plate capacitor. It is found that this distance is on the order of magnitude of Angstroms,
\end{abstract}


indicating that the measured electrical double layer capacitance is in fact the Stern layer capacitance.

\section{Keywords}

Electrical Double Layer, Stern Layer, Diffuse Layer, Nonionic Surfactant, Inverse Micelle, Electrical Impedance Spectroscopy 


\section{Introduction}

The stabilization of free electric charges in nonpolar media is rather difficult due to the Bjerrum length which is considerably larger than in polar media. ${ }^{1,2}$ The Bjerrum length is defined as $l_{B}=e^{2} /\left(4 \pi \varepsilon_{r} \varepsilon_{0} k_{B} T\right)$, where $e$ is the elementary charge, $\varepsilon_{r}$ is the relative permittivity of the medium, $\varepsilon_{0}$ is the permittivity of the vacuum, $k_{B}$ is the Boltzmann constant and $T$ is the temperature. ${ }^{2}$ It can be considered as the distance at which the electrostatic potential energy is equal to the thermal energy. ${ }^{2}$ In polar and nonpolar media, $l_{B}$ is roughly 1 and $30 \mathrm{~nm}$, respectively. If the electric charges are separated with a distance less than $l_{B}$, the electrostatic attraction overcomes the diffusion due to the thermal energy, which results in a recombination of opposite charges. Consequently, the existence of free charges in a nonpolar medium is considerably less likely compared to a polar medium. ${ }^{2,3}$ Nonetheless, in the presence of large entities, such as surfactant aggregations, free electric charges can be stabilized in nonpolar solvents. ${ }^{1-9}$ Surfactants are macromolecules which carry a considerable dipole moment. ${ }^{6}$ Above a certain concentration, they form aggregates which are commonly called inverse micelles. On the one hand, an inverse micelle can reduce the Bjerrum length by its larger permittivity. On the other hand, charges can be sterically-stabilized since relatively-large micelles keep them apart with a distance larger than the Bjerrum length. ${ }^{1,6}$ Moreover, inverse micelles form around traces of water and such a polar inverse micelle core also reduces the electrostatic interactions which facilitates the ionization. ${ }^{10}$ The possible origin of the ionizable species include impurities on the level of ppb, traces of fatty acids in the nonpolar solvent, as well as impurities in the surfactant along with traces of water. ${ }^{6}$ The stabilization of electric charges and their effects in nonpolar media are reported in numerous studies which are summarized in several comprehensive review articles. ${ }^{1-9}$

Despite the unfavorable nature of nonpolar solvents in terms of electrical charges, they have important respective applications including the prevention of flow electrification in oil industries, ${ }^{11,12}$ electrorheological fluids, ${ }^{13,14}$ electrophoretic displays, ${ }^{15-17}$ printer toners, ${ }^{18}$ 
and drug delivery. ${ }^{19,20}$ Still, the charge formation and interactions in nonpolar media are by far less understood compared to those in polar media. ${ }^{1,5}$ Likewise, it is unclear whether the conventional electrical double layer (EDL) theories can be applied to charged surfaces in contact with such systems. ${ }^{21}$ For example, since the charge content is usually much smaller than in a polar medium, the question of the minimum charge amount that is necessary to establish an EDL can be asked.

There are various experimental methodologies to study the charge formation and the EDL of dispersed and bulk solids in contact with nonpolar liquids; these include but are not limited to electrical conductivity measurements, ${ }^{6,10,22-28}$ electrophoretic mobility measurements, ${ }^{28-33}$ transient current measurements, ${ }^{34-41}$ or total internal reflection microscopy. ${ }^{42}$ Recently, Yezer et al. ${ }^{43,44}$ used electrical impedance spectroscopy (EIS) to study the EDL of mixtures of dodecane and the nonionic surfactants OLOA 11000, Span 80, and Span 85. This method can be favorably-used to determine the conductivity and the permittivity of the mixture as well as the capacitance of the EDL at the respective interface of an electrode. However, to conduct such experiments which resolve EDL effects, a setup with an extremely low electrode distance, typically on the order of magnitude of $10 \mu \mathrm{m}$, is necessary. For larger electrode distances, the required frequency range is below the resolution of common potentiostats. Yezer et al. established such a low electrode distance by using dispersed silica microparticles as spacers. ${ }^{43,44}$ However, such microparticles also acquire EDLs and therefore form increased conductivity pathways between the electrodes. These "short circuits" disturb the local potential distribution. Likewise, the particle volume displaces liquid and silica has a two times higher permittivity compared to dodecane. Thus, setups with narrow electrode distances which contain particle-free liquids are more desirable.

The first objective of our study is to characterize the charge formation of a nonpolar medium in the presence of a nonionic surfactant. The nonionic surfactant is chosen to 
ensure that it acts as a (steric) stabilizer rather than a dissociable charge controlling agent. In contrast to the work of others, we take the viscosity change due to the surfactant addition into account. The other main novelty of this work is that we resolve the EDL structure of planar electrodes in contact with such nonpolar mixtures. For this purpose, we apply EIS measurements in two custom-made cells which we discuss in the Experimental Section. In the Results and Discussion Section, we present water content, viscosity and inverse micelle size, along with typical impedance spectra that we measure. We introduce appropriate equivalent circuits necessary for the spectra interpretation. From regression of the equivalent circuit, we infer the properties of the mixtures and the characteristics of the EDL in terms of Stern and diffuse layers. Finally, this article is concluded with a summary section.

\section{Experimental Section}

In this section, we give information on the chemicals and methods that are used in this work.

\section{Chemicals}

Dodecane $\left(\mathrm{C}_{12} \mathrm{H}_{26}\right)$ with a permittivity of 2 is used as the nonpolar solvent (mixture of isomers, ACROS Organics, Germany). The nonionic surfactant sorbitan monooleate $\left(\mathrm{C}_{24} \mathrm{H}_{44} \mathrm{O}_{6}\right)$, known commercially as Span 80 (TCI America, Portland, OR, U.S.A.), is used as the solute. Span 80 has a 16 carbon chain tail, a polar sorbitan head, and a permittivity of 4.7. ${ }^{24}$ The critical micelle concentration (CMC) of Span 80 in dodecane is reported to be $1.9 \cdot 10^{-5} \mathrm{M}^{45}$ In order to prepare the sample mixtures, Span 80 is dissolved in dodecane at concentrations varying from $1 \mathrm{mM}$ to $1 \mathrm{M}$. The chemicals are used as received from the supplier without further purification. All glassware used in the preparation of solutions are cleaned thoroughly with DI water and isopropyl alcohol and placed in an oven to dry overnight. Humidity of 
the environment is not controlled but each solution is stored in a sealed bottle when not in use to prevent water uptake from the environment.

\section{Sample Characterizations}

The water content of the samples are measured with a Karl Fischer (KF) titration Coulometer KF 756 (Metrohm, Switzerland). A sample volume of 1 - $3 \mathrm{~mL}$ is injected into the system for each test. Hydranal ${ }^{T M}$-Coulomat AG (Honeywell Fluka, Germany) is used as the analyte solution.

To measure the size of the inverse micelles, we employ dynamic light scattering (DLS) using a Zetasizer Nano ZS (Malvern, U.K.). The angle of detection is set to be $173^{\circ}$ in order to maximize the detection of the light scattered by the very small inverse micelles. The value of the refractive indices of dodecane and Span 80 are set to be 1.421 and 1.48, respectively. An absorption value of 0.001 is used as the minimum possible value for the instrument since the samples are transparent. The samples are placed in a glass cuvette, type PCS 1115 (Starna, U.K.) and measurements are carried out at an instrument temperature of $25{ }^{\circ} \mathrm{C}$. Viscosity of the samples are measured at room temperature with a falling ball viscometer (Gilmont Instruments, Barrington, IL, U.S.A.).

\section{Electrical Impedance Spectroscopy}

EIS measurements are performed with an Autolab PGSTAT302N potentiostat/galvanostat (Metrohm Autolab B.V., Netherlands). Sinusoidal excitation signals with two different amplitudes of 10 and $50 \mathrm{mV}$ are used in this work. Comparison shows that there are almost no differences between the results; however, an amplitude of $50 \mathrm{mV}$ reduces the noise in the spectra. The frequency range of the experiments is adapted to the two different measure-

ment cells (details given below). Each measurement is conducted at room temperature with 
at least three replicates. The regression of the impedance spectra is performed with the software NOVA 1.11 (Metrohm Autolab B.V., Netherlands).

We utilize two different measurement cells since this allows for a convenient detection of the bulk and the EDL phenomena that we are interested in. Figure 1 (left) shows a sketch of cell 1 which is comprised of two circular indium tin oxide (ITO) electrodes that are coated on a boro-aluminosilicate glass wafer (non-alkaline grade, University Wafer, U.S.A.). The ITO electrodes have a diameter of $50 \mathrm{~mm}$, a thickness of $0.7 \mathrm{~mm}$ and a surface resistivity of around $10 \Omega$ sq. Two custom-made holders separate the electrodes with a $2 \mathrm{~mm}$ gap. The holder components are printed using a Miicraft + 3D printer (Miicraft, Hsinchu, Taiwan). The wires are physically in contact with the electrodes through an opening in each holder. To perform the measurements, the assembled cell is placed in a petri dish which is filled with the liquid sample.

\section{Cell 1}

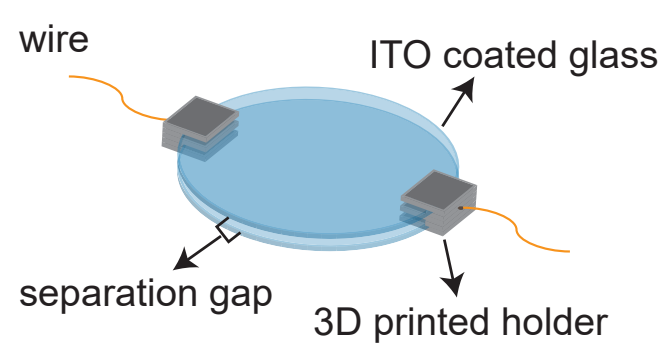

Cell 2

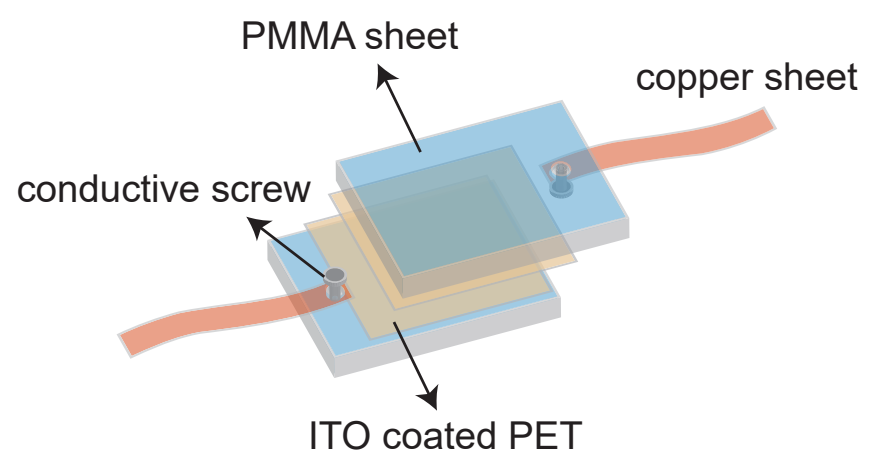

Figure 1: Sketch of the two different cell designs employed for the EIS measurements.

Figure 1 (right) shows the design of cell 2. It is composed of two ITO electrodes coated on a polyethylene terephthalate (PET) film (Aldrich Chemistry, St. Louis, MO, U.S.A.). The electrodes have a thickness of $127 \mu \mathrm{m}$ and a surface resistivity of around $10 \Omega / \mathrm{sq}$. Each electrode is attached to a PMMA sheet. A metallic screw is placed through a hole in the PMMA sheet and connects the electrode to a copper sheet that serves as external electrical 
contact. First, the surface of the bottom electrode is covered with a liquid film of the sample. Then, the other electrode is placed on top of the wetted lower electrode which establishes a electrode distance without using any spacer.

The surface area and electrode distance of cell 1 is known. However, this electrode distance is relatively large, resulting in a very high ohmic resistance of the liquid, which suppresses any EDL effect in the impedance spectra. The wetted surface area of cell 2 can be directly measured and usually varies in the range of $21-23 \mathrm{~cm}^{2}$. The electrode distance in cell 2 is the thickness of the liquid film. It is extremely small, which promotes the characteristics of the EDL in the impedance spectra, but its exact value is unknown. Nevertheless, both cells allow for the measurement of the bulk properties of the sample and we use results from cell 1 to infer the electrode distance in cell 2. In detail, we use EIS measurements in the frequency range of $1 \mathrm{MHz}$ to $100 \mathrm{~Hz}$ in cell 1 to measure the capacitance which is then used to calculate the (bulk) permittivity of the sample. Subsequently, EIS

measurements are performed in a frequency range of $1 \mathrm{MHz}$ to $10 \mathrm{mHz}$ in cell 2 . From the measured liquid bulk capacitance, and the bulk permittivity from the cell 1 measurements, the electrode distance of cell 2 can be determined. Once the electrode distance is known, the impedance spectra can be evaluated in terms of the sample conductivity and EDL properties at the electrode interface.

\section{Results and Discussion}

In this section, we first report the results of the KF titration and viscosity of the samples, as well as the inverse micelle size measurements. These data are required for further evaluation of the phenomena that we investigate. Then, we present impedance spectra of exemplary samples measured in cell 1 and 2. Based on these spectra, we discuss the sample permit- 
tivity and conductivity accompanied with further discussion about diffusivity, charge carrier concentration as well as the EDL characteristics.

\section{Water Content}

The water content of the samples as a result of the Span 80 concentration in dodecane is shown in Figure 2. We observe a constant increase in the water content when we add the

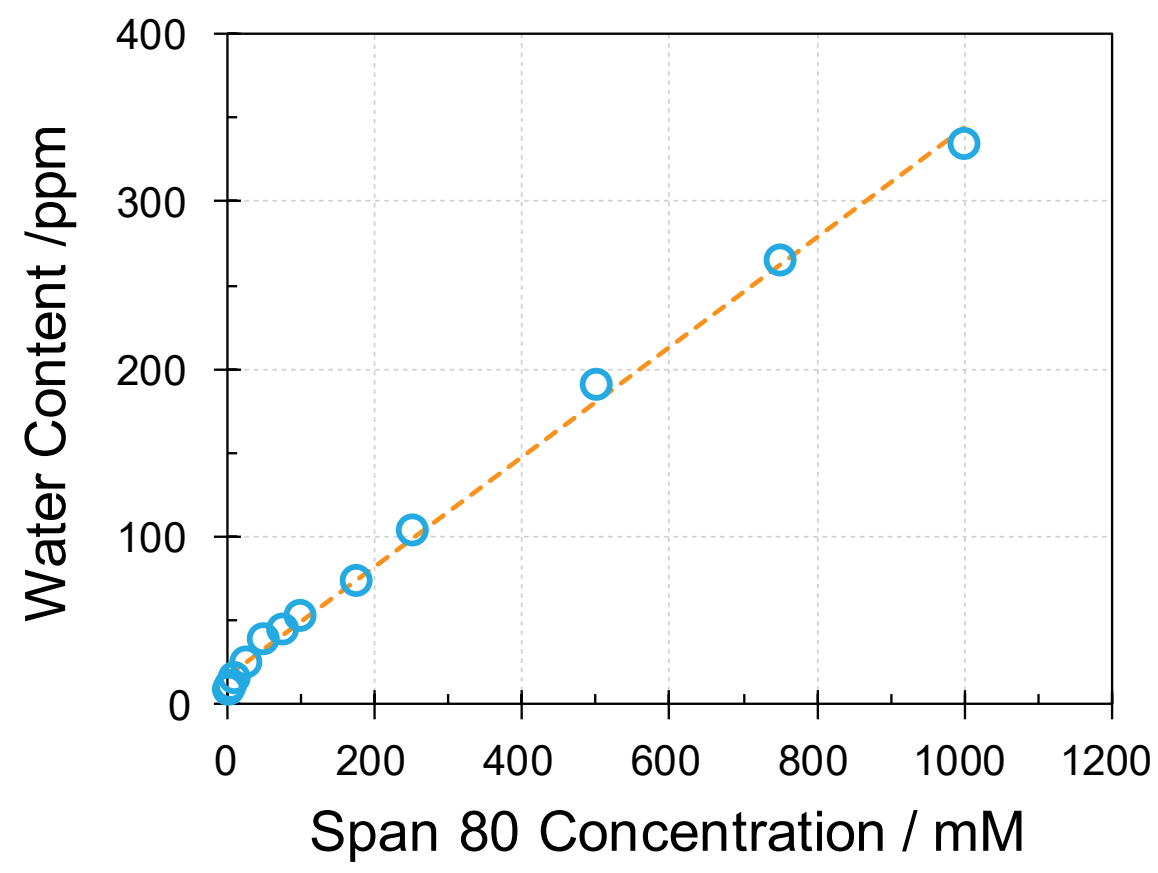

Figure 2: Sample water content versus Span 80 concentration in dodecane. The standard deviation of the measurements is smaller than the size of the symbols.

surfactant. This increase is linear with a very high coefficient of determination of $R^{2}=0.997$. A regression results in

$$
\frac{c_{w}}{\mathrm{ppm}}=0.3268 \frac{c_{s}}{\mathrm{mM}}+17
$$

where $c_{w}$ and $c_{s}$ are the water and Span 80 concentration in the sample, respectively. This linearity indicates that the water in the samples originates from the surfactant. The maximum water content, observed for the sample with the maximum surfactant concentration, is $335 \pm 8 \mathrm{ppm}$, which corresponds to less than $0.04 \mathrm{wt} \%$. The presence of water facilitates, ${ }^{1}$ 
or is even necessary, ${ }^{2,7}$ for the formation of the inverse micelles. In addition, water traces, or any other impurity, was suggested as the possible source of ionizable species in the solutions of a nonionic surfactant in nonpolar media. ${ }^{2,7}$ Ionic species can also dissociate in water that is present in the system. Therefore, any addition of water to a nonpolar system, with or without surfactants, increases its conductivity. ${ }^{3}$

\section{Viscosity}

The sample viscosity is required for the DLS measurements and for the estimation of the charge carrier concentration. Figure 3 shows the viscosity for different samples of Span 80 dissolved in dodecane. The viscosity increases from $1.3 \mathrm{mPa}$.s for pure dodecane to 16.4 mPa.s for the sample containing $1000 \mathrm{mM}$ of Span 80. Above concentrations of around 500 $\mathrm{mM}$, the addition of surfactant considerably increases the viscosity. A suitable correlation between viscosity $\eta$ and surfactant concentration is

$$
\frac{\eta}{\mathrm{mPa} . \mathrm{s}}=2 \cdot 10^{-8}\left(\frac{c_{s}}{\mathrm{mM}}\right)^{3}-1 \cdot 10^{-5}\left(\frac{c_{s}}{\mathrm{mM}}\right)^{2}+4.3 \cdot 10^{-3} \frac{c_{s}}{\mathrm{mM}}+1.3
$$

having a very high coefficient of determination of $R^{2}=0.999$. In addition, Dukhin et al. ${ }^{46}$ showed that the viscosity of Span 80 in toluene follows a semi-empirical mixing rule according to

$$
\ln \left(\eta_{m} V_{m}\right)=x_{1} \ln \left(\eta_{1} V_{1}\right)+x_{2} \ln \left(\eta_{2} V_{2}\right)+x_{1} x_{2} \frac{E_{12}}{R T}
$$

where $\eta_{i}, V_{i}$, and $x_{i}$ are viscosity, molar volume and mole fraction of mixture $m$ or component 1,2 , respectively, $E_{12}$ is the molecular interaction energy, $R$ is the universal gas constant and $T$ is the temperature. Applying Equation (3) to fit our viscosity measurements results in a slightly lower but still excellent coefficient of determination of $R^{2}=0.998$. The molecular interaction energy is then obtained to be $-10.9 \pm 0.2 \mathrm{~kJ}$ which is comparable to 


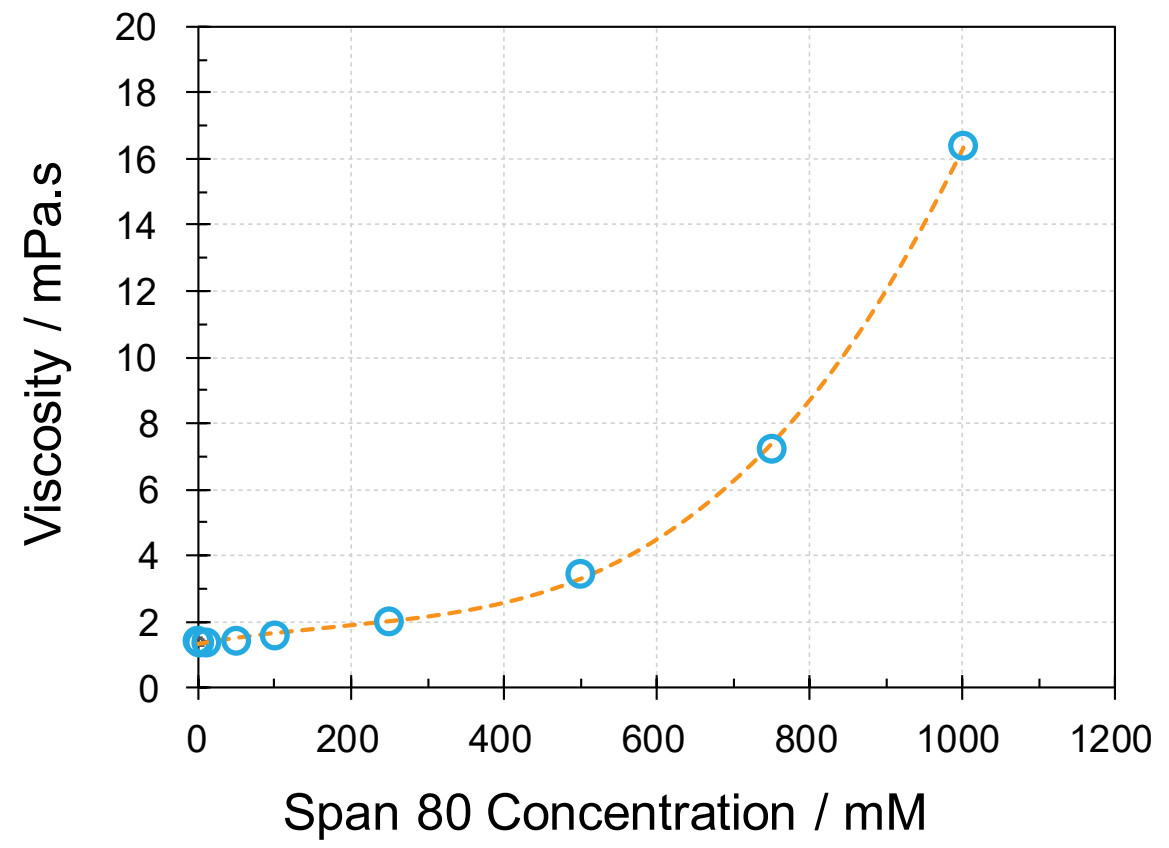

Figure 3: Sample viscosity versus Span 80 concentration in dodecane. The standard deviation of the measurements is smaller than the size of the symbols.

the $-21.3 \mathrm{~kJ}$ which was reported by Dukhin et al. for mixtures of Span 80 in toluene.

\section{Inverse Micelle Size}

The (equivalent) hydrodynamic diameters $d_{h}$ of the inverse micelles in a Span 80 concentration range of 5 to $250 \mathrm{mM}$ in dodecane are shown in Figure 4 . Here, we report the average diameter based on the intensity distribution of the DLS measurements. It should be noted that for concentrations above $250 \mathrm{mM}$, we could not obtain reliable results. It seems that the size somewhat decreases with increasing surfactant concentration; the drop is lower at the higher surfactant concentrations. At the lowest concentration of $5 \mathrm{mM}$, the average size is $8 \pm 4 \mathrm{~nm}$. A smaller average size of $4 \pm 1 \mathrm{~nm}$ is measured for the sample with 250 mM. These results are in good agreement with those reported in ref. ${ }^{47}$ The relatively large standard deviations suggest a broader distribution of inverse micelle sizes within the sample. Thus, we cannot be sure whether the average micelle size really decreases since the change is 
more or less within the standard deviations of the measurements. In fact, most of the commercial surfactants are mixtures of various molecular structures which can produce a range of inverse micellar configurations with different sizes. ${ }^{43}$ The large standard deviation is also in agreement with the discussion provided in ref. ${ }^{6}$ regarding the fact that inverse micelles, especially made from nonionic surfactants, and with different charges do not have equal sizes. In addition, Dukhin et al. ${ }^{48}$ discussed that a charged inverse micelles should be smaller than ta neutral inverse micelles due to the interaction between polar head and central charge. In the DLS measurements, we indeed measure the size of uncharged inverse micelles as they are abundant in the sample. Moreover, it was reported that inverse micelles made from Span 80 are rather not spherical. ${ }^{47,49}$ This is not a problem since the equivalent diameter that we measure is that of a sphere which behaves like a (non-spherical) entity. Nonetheless, for the estimations that we perform below, we consider the inverse micelles to have spherical shape and we choose an average equivalent diameter of $5 \mathrm{~nm}$ over all concentrations. This value is also in good agreement with the diameter of $4 \mathrm{~nm}$ reported by Karvar et al. ${ }^{37}$ which is derived from transient current measurements.

\section{Electrical Impedance Spectroscopy}

EIS measurements of mixtures of Span 80 and dodecane are carried out using both cell 1 and cell 2. Figure 5a shows typical spectra of samples with low, intermediate and high concentrations measured in cell 1. For all concentrations, there is (a segment of) a semicircle which represents the capacitive and resistive behavior of the liquid bulk along with the resistive behavior of the electrodes. The higher the surfactant concentration, the more of the semicircle perimeter can be seen in the spectrum. The diameter of the semicircle decreases

with increasing surfactant concentration. Indeed, the difference between the two intersects of the spectrum with the $\mathrm{x}$-axis (real part of the impedance) corresponds to the ohmic resistance 


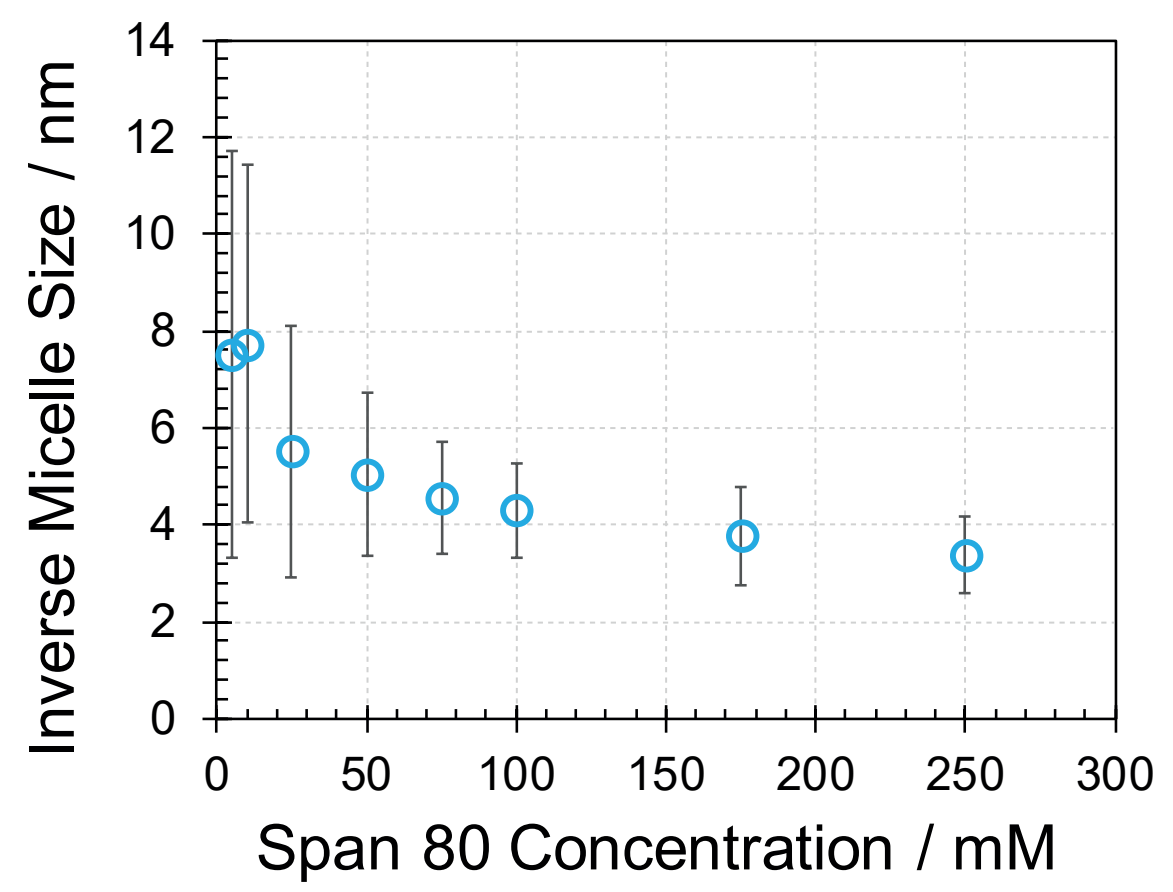

Figure 4: Average inverse micelle size (equivalent hydrodynamic diameter) versus Span 80 concentration in dodecane.

of the sample. This behavior is related to the conductivity increase even though we add a nonionic surfactant. The spectra obtained using cell 1 are fitted (solid line) to the equivalent $\mathrm{R}(\mathrm{RC})$ circuit shown as insert in Figure 5a. In this circuit, $R_{e}$ is the ohmic resistance of the electrodes while $R_{b}$ and $C_{b}$ are the ohmic resistance and the capacitance of the liquid bulk, respectively. The geometry of cell 1 is known which enables us to calculate the permittivity of each sample from the measured capacitance (reported below).

Next, we present results from EIS measurements using cell 2. The impedance spectra of samples containing 25, 100 and $1000 \mathrm{mM}$ Span 80 are shown in Figure 5b-d, respectively. There are three distinguishable regions in these spectra: (i) the high frequency semicircle related to the liquid bulk properties; (ii) a mid-range frequency distributed response which arises from the transport effects; (iii) and another tail-like segment of a low frequency EDL semicircle. We note that for higher surfactant concentrations, the high-frequency semicircle and the EDL segment become smaller and more pronounced, respectively. In addition, the mid-range frequency distributed region (the influence of transport effects) becomes smaller 

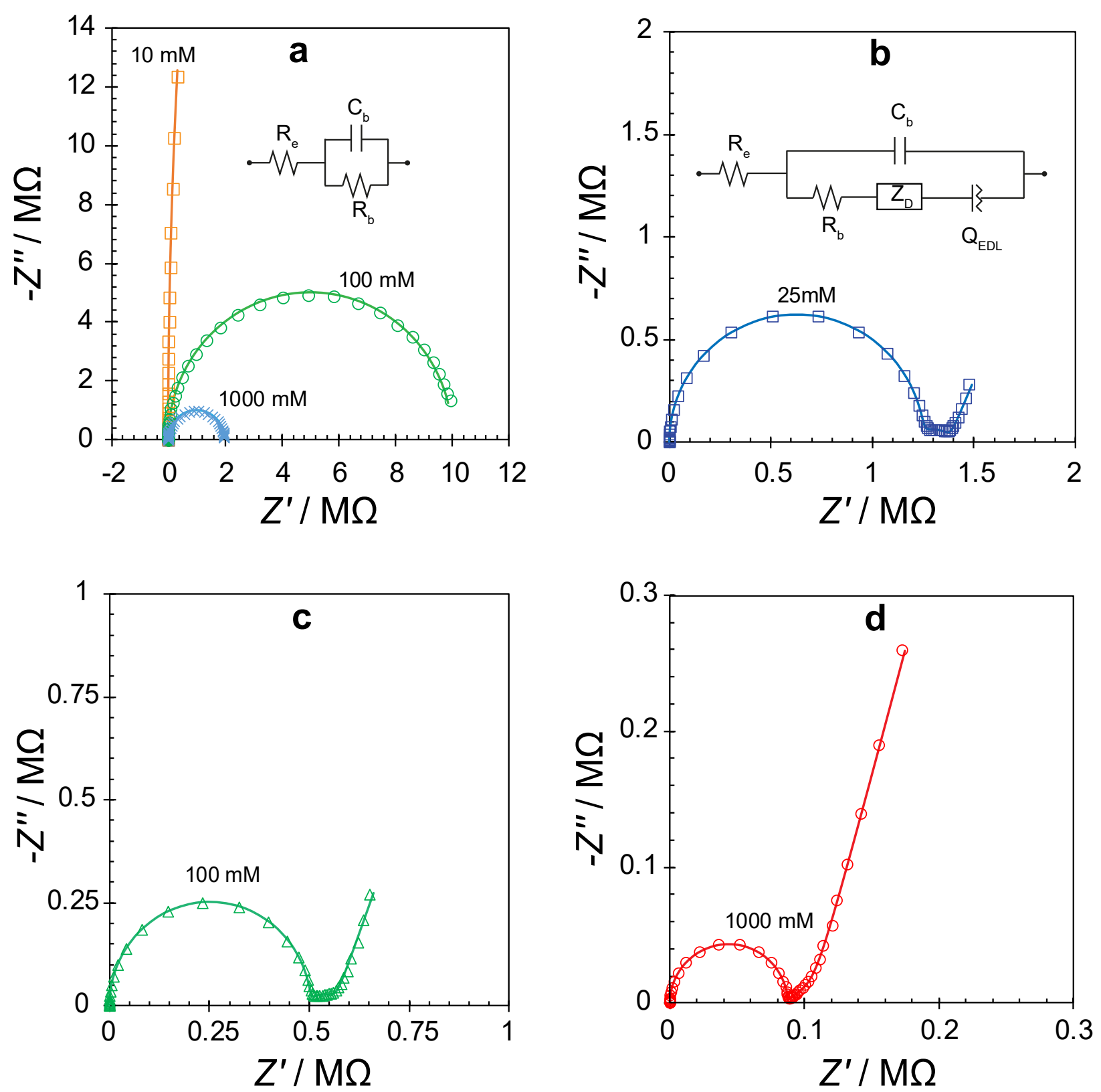

Figure 5: Typical EIS spectra of various concentration of Span 80 in dodecane measured in cell 1 or 2: a) 10, 100 and $1000 \mathrm{mM}$ Span 80 measured in cell 1; b) $25 \mathrm{mM}$ Span 80 measured in cell 2; c) $100 \mathrm{mM}$ Span 80 measured in cell 2; d) $1000 \mathrm{mM}$ Span 80 measured in cell 2. 
as the concentration increases.

All impedance spectra that are measured with cell 2, are fitted (solid lines) to the equivalent circuit shown as the inset in Figure 5b. This circuit is a simplified form of an

equivalent circuit that is adequate for the interpretation of most electrochemical spectra. ${ }^{50,51}$ Here, $R_{e}, C_{b}$ and $R_{b}$ have the same definition as in the liquid bulk circuit used in Figure 5 a. The impedance $Z_{D}$ capturing the transport effects is a distributed element reflecting the diffusion in the system. When mobility and size of a diffusive species are very different, the respective impedance does not have a single time constant. ${ }^{50-53}$ As mentioned above, the size of the inverse micelles in our system features a rather large standard deviation. This indicates a certain size and mobility distribution which results in the appearance of the midrange frequency distributed response in the spectra. We observe the best regression quality if $Z_{D}$ is described by a parallel combination of a resistor $R_{d}$ and a Warburg element $W_{d}$. The EDL tail is captured by a constant phase element (CPE) $Q_{E D L}$ which usually gives a better regression of the capacitive EDL effect since it accounts for the non-ideality of the

electrode surface. ${ }^{54-56}$ The impedance of a CPE is defined as $Z_{Q}=1 / Q_{0}(j \omega)^{n}$ where $Q_{0}$ is the CPE coefficient, $n$ is an empirical shape factor with a value between 0 and $1, j$ is the imaginary unit and $\omega$ is the angular frequency. Note that the equivalent circuit in Figure 5b includes only one element for the transport and one for the EDL effects since both electrodes in the cell are equal. The regression results for all spectra are reported in the Supporting Information. The respective elements of the equivalent circuits are used to compute the liquid bulk conductivity as well as the capacitance of the EDL.

\section{Permittivity}

The permittivity of the samples are derived from the impedance spectra measured with cell 1. In detail, the liquid bulk capacitance $C_{b}$ is obtained from the regression of the equivalent circuit shown in Figure 5a to the measured spectra. Knowing the electrode distance $d$ and 
the surface area of the electrodes $A$, the relative permittivity corresponds to

$$
\varepsilon_{r}=\frac{C_{b} d}{A \varepsilon_{0}},
$$

where $\varepsilon_{0}$ is the permittivity of the vacuum. The results for $\varepsilon_{r}$ versus the surfactant concentration in dodecane are shown in Figure 6. We observe a linear correlation between the relative permittivity and the surfactant concentration. A respective regression results in

$$
\varepsilon_{r}=9 \cdot 10^{-4} \frac{c_{s}}{\mathrm{mM}}+2.0
$$

Surfactant molecules carry a substantial dipole moment; ${ }^{6}$ this explains the observed increase in the sample permittivity when surfactant is added.

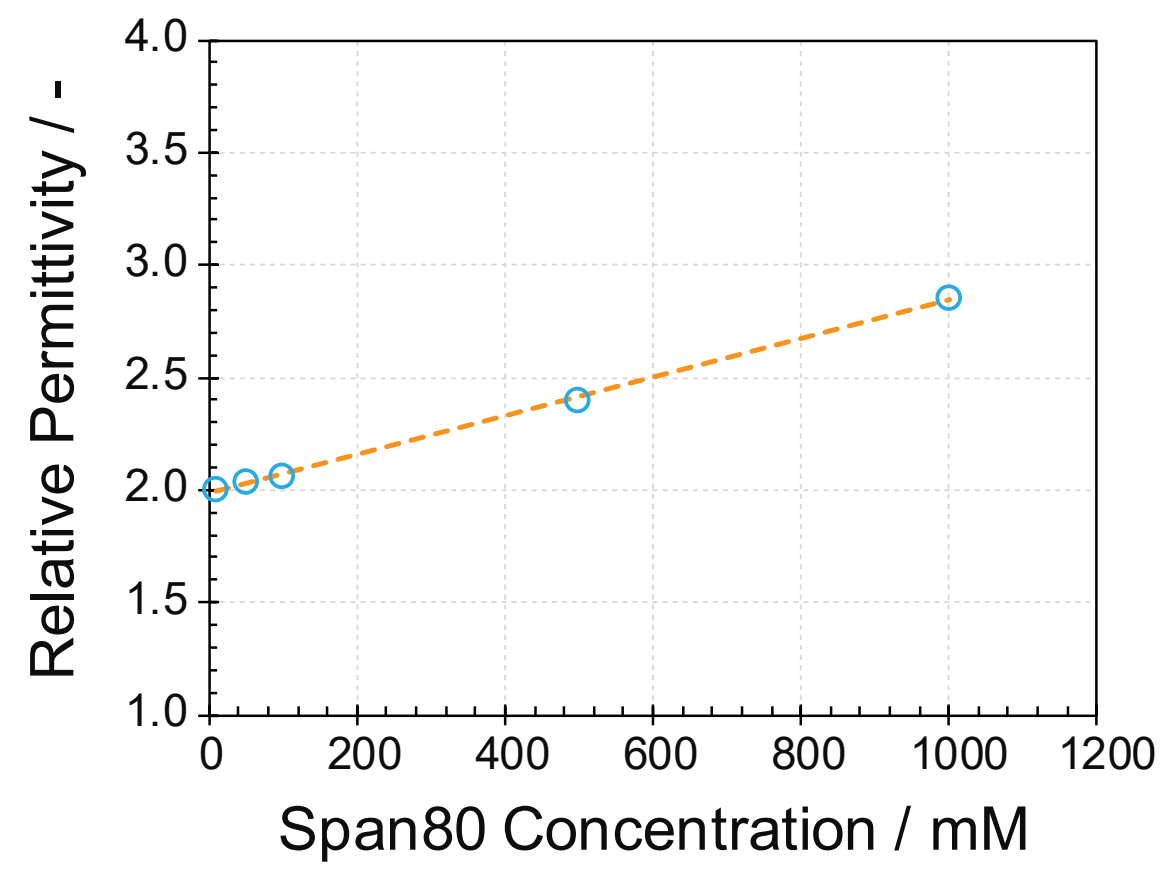

Figure 6: Relative permittivity of samples with various concentrations of Span 80 in dodecane. The standard deviation of the measurements is smaller than the size of the symbols. The dashed line shows the linear regression. 


\section{Conductivity}

The liquid bulk resistance $R_{b}$ that can be extracted from an EIS spectrum is very informative with respect to the formation and interactions of the charge carriers in a nonpolar medium. This resistance can be converted to the medium conductivity if the cell geometry is known. As discussed above, we use the permittivity results from cell 1 along with Equation (4) to calculate the electrode distance in cell 2. Generally, this electrode distance is in the range of 50 to $80 \mu \mathrm{m}$ and we can calculate the sample conductivity according to

$$
\sigma=\frac{d}{A R_{b}} .
$$

The so-inferred conductivity results are plotted in Figure 7 on a log-log scale since a respective correlation can be conveniently described with a power law. We observe that the medium

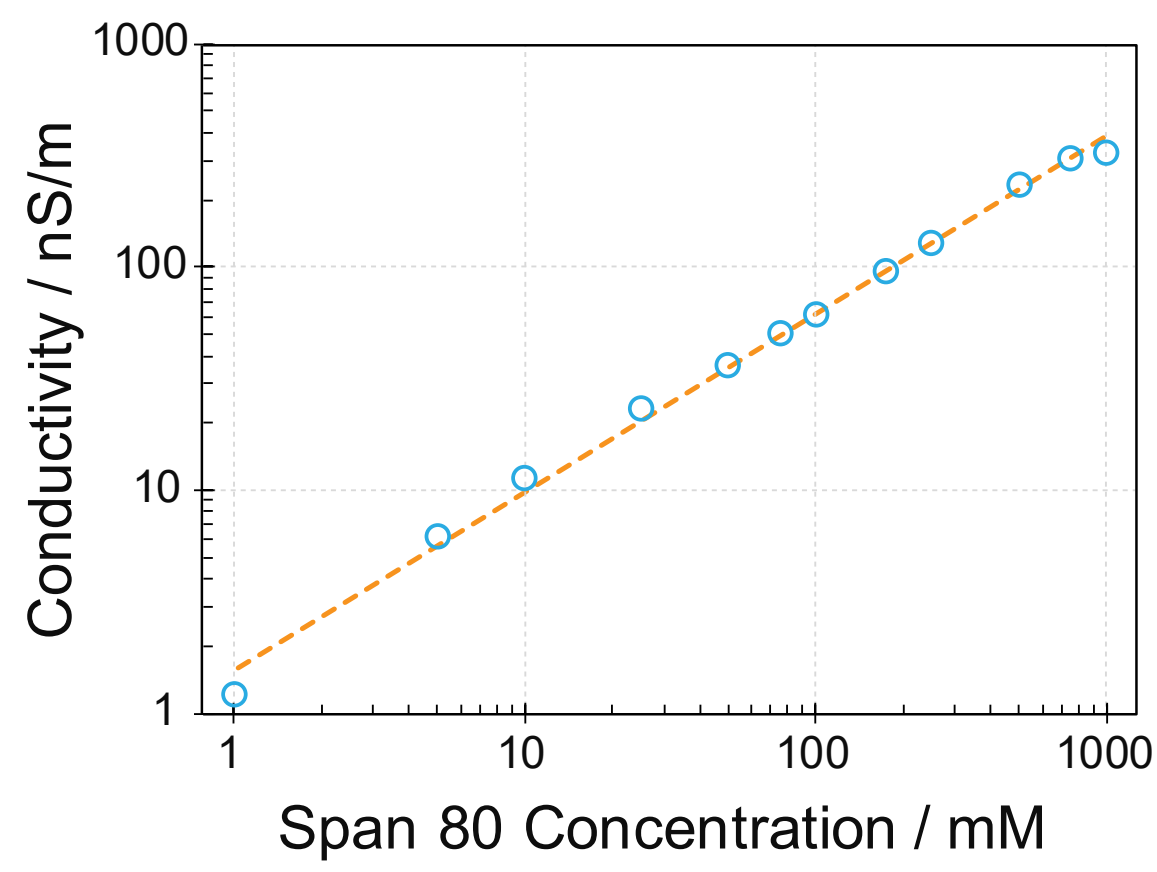

Figure 7: Electric conductivity of samples with various concentrations of Span 80 in dodecane. The standard deviation of the measurements is smaller than the size of the symbols. The dashed line shows the power law regression.

conductivity $\sigma$ increases with the addition of the nonionic Span 80 surfactant. The regression 
of the data to

$$
\frac{\sigma}{\mathrm{nS} / \mathrm{m}}=1.566\left(\frac{c_{s}}{\mathrm{mM}}\right)^{0.8}
$$

results in a very high coefficient of determination of $R^{2}=0.996$. There are two well-known models for describing the charge generation in nonpolar media in the presence of surfactants; i.e., the disproportionation and the dissociation model. ${ }^{6}$ In the disproportionation model, it is assumed that two neutral inverse micelles collide, exchange electric charge, and form two oppositely-charged inverse micelles. ${ }^{3,4,6,23,57}$ If there is neither a change in the viscosity nor in the inverse micelle size, this model predicts a linear correlation between the medium conductivity and the surfactant concentration. ${ }^{6,31}$ The disproportionation model does not eliminate the possibility of the existence of the neutral ion-pairs but predicts there is no influence of the ion-pairs on the conductivity. ${ }^{9,58}$ In contrast, the dissociation model ${ }^{6,59-63}$ assumes that a neutral entity, such as molecules or ion-pairs, can dissociate into two oppositely charged species. Below a critical concentration, which corresponds to a inverse dissociation constant, this model results in a quadratic dependency between medium conductivity and surfactant concentration. At higher concentrations, but still below the critical concentration, the quadratic dependency results in a decrease of the conductivity slope. This would imply the recombination of dissociated species into neutral ion-pairs. Above the critical concentration, the theory predicts a square root dependency for the conductivity. ${ }^{6}$

For a reasonable prediction of the conductivity, we use the common correlation for diluted electrolytes

$$
\sigma=F \sum z_{i} c_{i} \lambda_{i}=F^{2} \sum \frac{z_{i}^{2} c_{i} D_{i}}{R T}
$$

where $\lambda_{i}, c_{i}, z_{i}$, and $D_{i}$, are the mobility, concentration, valency and diffusivity of the ionic species $i, F$ is the Faraday constant, $R$ is the universal gas constant and $T$ is the temperature. 
The diffusivity can be approximated with the Stokes-Einstein equation

$$
D=\frac{R T}{3 \pi N_{a v} \eta d_{h}}
$$

where $N_{a v}$ is the Avogadro number. We further define $\chi$ as the charged inverse micelles fraction and $N_{a g}$ as the aggregation number of an inverse micelle. We also do the reasonable assumption that positively- and negatively-charged inverse micelles have the same size and diffusivity. Hence, we can express the correlation between conductivity and surfactant concentration according to

$$
\sigma=\frac{(z F)^{2} \chi c_{s}}{3 \pi N_{a v} N_{a g} \eta d_{h}}
$$

For the identification of the charge formation model, the fact that the medium viscosity can considerably change with the surfactant content is usually disregarded. If we consider that $z$, $\chi, N_{a g}$, and $d_{h}$, are constant, Equation (10) reveals that the viscosity-adjusted conductivity $\sigma^{\prime}=\sigma \eta$ is a linear function of the surfactant concentration. We test this hypothesis in Figure 8. A linear dependency between $\sigma^{\prime}$ and $c_{s}$ is observed up to around $250 \mathrm{mM}$. This indicates that in this concentration range, the charge formation takes place according to the disproportionation model. At higher concentrations than $250 \mathrm{mM}$, higher viscosity adjustedconductivities are measured than predicted. This could be related to interactions between the increased number of inverse micelles which may increase the fraction of charged inverse micelles or lower the aggregation number which results in smaller and more mobile inverse micelles. To summarize, our viscosity adjusted conductivity approach clarifies that the (drastic) change in the conductivity slope in Figure 7 (if plotted on a linear scale), is related to the viscosity change of the medium and not to the formation of ion-pairs. This is also supported by the fact that the nonlinear behavior of $\sigma^{\prime}$ at higher concentrations has an increasing slope. The slope as a result of the dissociation model is expected to be decreasing. In conclusion, there is no indication for the validity of the dissociation model while the behavior up to $250 \mathrm{mM}$ surfactant concentration follows the disproportionation model. 


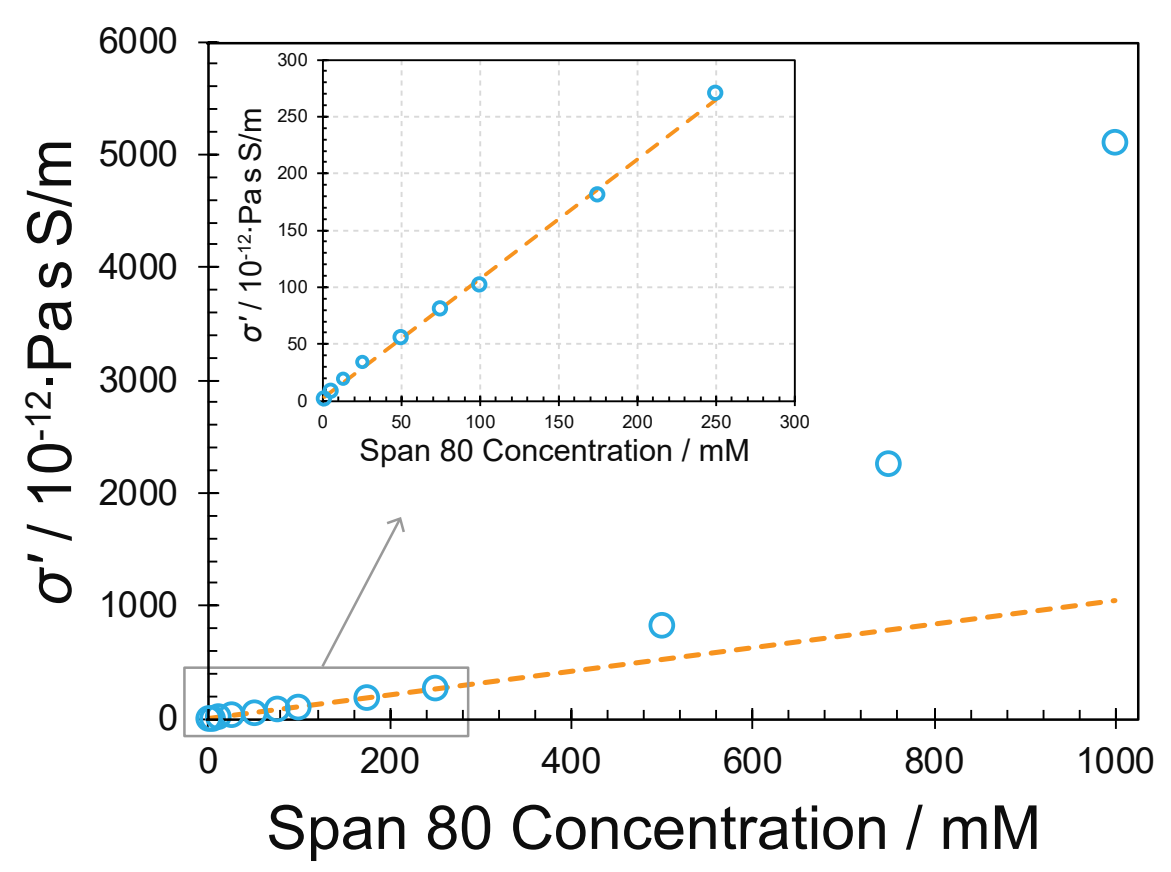

Figure 8: Viscosity-adjusted conductivity for various concentrations of Span 80 in dodecane. The dashed line results from the linear regression of the data up to $250 \mathrm{mM}$.

We use the conductivity data to estimate the concentration of charged inverse micelles in our samples. Note that the following analysis is of rather qualitative nature and the results are expected to be on the right order of magnitude and not necessarily precise values. Here, we assume that the inverse micelles have an equivalent hydrodynamic diameter of 5 nm. Likewise, a charged inverse micelle is not significantly smaller and carries an elementary charge $(z= \pm 1)$. We limit the concentration range of interest to the linear regime of the viscosity-adjusted conductivity. The average diffusivity of the inverse micelles in this range of concentrations is calculated using Equation (9); we arrive in $5.3 \cdot 10^{-11} \mathrm{~m}^{2} / \mathrm{s}$. This value is similar to the diffusivities reported in the literature for various inverse micelles in nonpolar media. ${ }^{24,43,44,57,64}$ The aggregation number of Span 80 in dodecane is reported to be between 3 to 20 for the concentration range. ${ }^{47}$ Hence, we assume an aggregation number of 10 . Using these material properties along with Equation (10), we calculate $\chi \simeq 3.2 \cdot 10^{-5}$ as the average fraction of charged inverse micelles. This value is very similar to those reported for the ionic aerosol-OT $(\mathrm{AOT})\left(\chi=1.2 \cdot 10^{-5}\right),{ }^{22}$ nonionic OLOA $371\left(\chi=2 \cdot 10^{-5}\right),{ }^{23}$ nonionic 
OLOA $11000\left(\chi=4 \cdot 10^{-5}\right),{ }^{10}$ ionic $\operatorname{AOT}\left(\chi=1.5 \cdot 10^{-5}\right)$, ionic zirconyl 2-ethyl hexanoate $\left(\chi=2.7 \cdot 10^{-5}\right),{ }^{25}$ ionic Na-AOT $\left(\chi=2.2 \cdot 10^{-5}\right)$ and ionic $\operatorname{Li}-\mathrm{AOT}\left(\chi=2.2 \cdot 10^{-5}\right),{ }^{65}$ all in dodecane.

The knowledge of the amount of charged inverse micelles allows us to compute the ionic strength of the medium according to $I=\frac{\chi c_{s}}{2 N_{a g}}$. This in turn allows the determination of the (approximate) thickness of the EDL; i.e., the Debye length

$$
l_{D}=\left(\frac{\varepsilon_{r} \varepsilon_{0} R T}{2 F^{2} I}\right)^{1 / 2}
$$

We plot the computed Debye length versus the ionic strength as well as versus the surfactant concentration in Figure 9. The Debye length decreases from approximately $250 \mathrm{~nm}$ to 80 $\mathrm{nm}$ as the concentration increases from 25 to $250 \mathrm{mM}$. Regression of the data reveals that the calculated Debye length changes according to $l_{D} \propto c_{s}^{-0.43}$. This minor deviation from the inverse square root dependency is related to the changing medium permittivity due to surfactant addition which is included in our data evaluation but not in the solution of the Poisson-Boltzmann equation. In the next section, we present the measured EDL capacitances. Based on this, we calculate the thickness of an equivalent parallel-plate capacitor and compare it to the Debye length results presented here.

\section{Electrical Double Layer Capacitance}

From the values of the CPE, obtained from the regression of the EIS spectra measured in cell 2 to the equivalent circuit, we can calculate the equivalent EDL capacitance $C_{E D L}$. Here, we follow Burg et al. ${ }^{66,67}$ and use their equation for blocking electrodes with a planar 


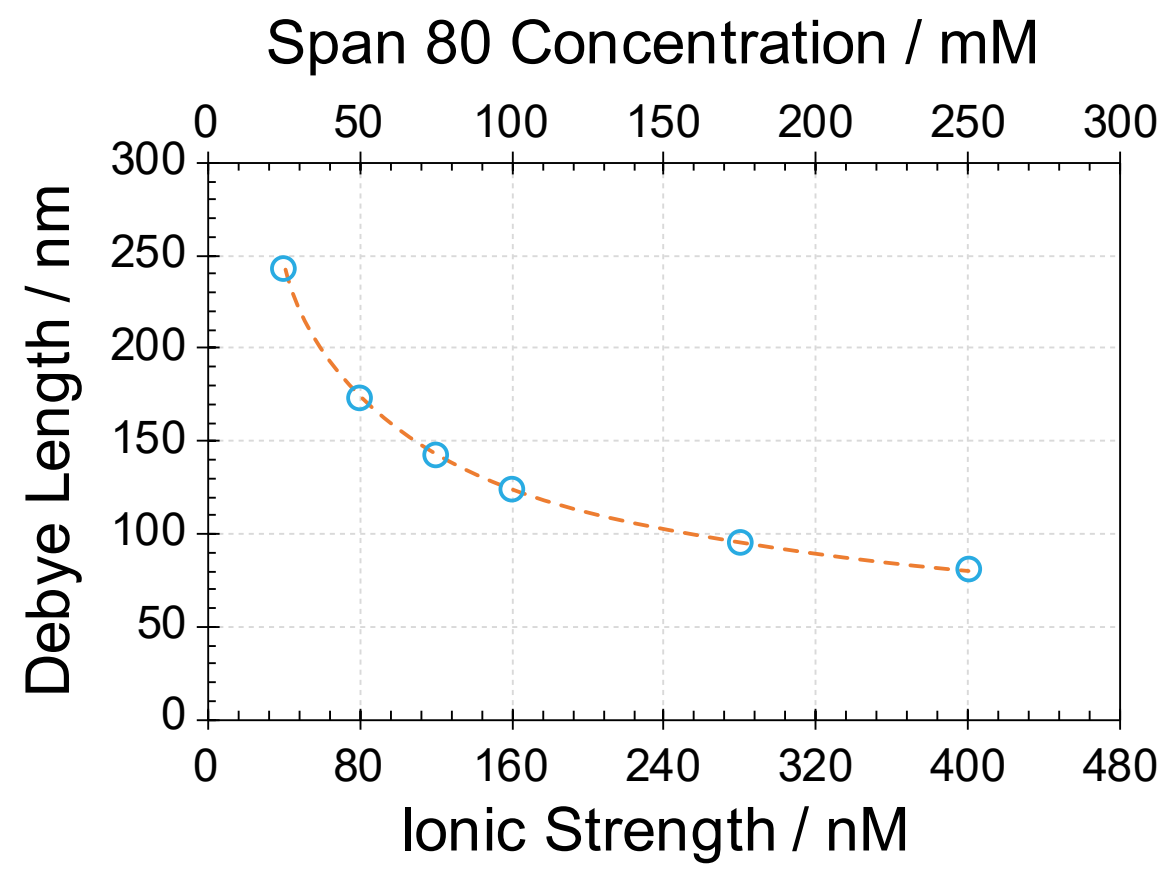

Figure 9: Debye length versus ionic strength and Span 80 concentration. The dashed line shows the proportionality according to $l_{D} \propto c_{s}^{-0.43}$.

distribution of time constants. It is

$$
C_{E D L}=2 Q_{0}^{1 / n} R_{s}^{\frac{1-n}{n}},
$$

where $R_{s}$ is the resistance in series with the CPE. In our system, it is $R_{s}=R_{e}+R_{b}+R_{d}$. The factor of 2 in Equation (12) is added since we have two equal electrodes but use only one CPE to capture their effects. The specific equivalent capacitances (triangular symbols) for various concentrations of Span 80 are plotted in Figure 10. It can be seen that, despite the surfactant concentration change over order of magnitudes, the equivalent capacitance remains on the same order of magnitude. In detail, there is a slight decrease from around 7 to $4 \mu \mathrm{F} / \mathrm{cm}^{2}$ when the Span 80 concentration increases from 10 to $1000 \mathrm{mM}$. This surfactant concentration range corresponds to an ionic strength range of around 20 to $2000 \mathrm{nM}$, when we use the parameters of the charged micelles also for the concentrations above $250 \mathrm{mM}$. Since in a nonpolar medium the relative permittivity is not significantly impacted by the electric 


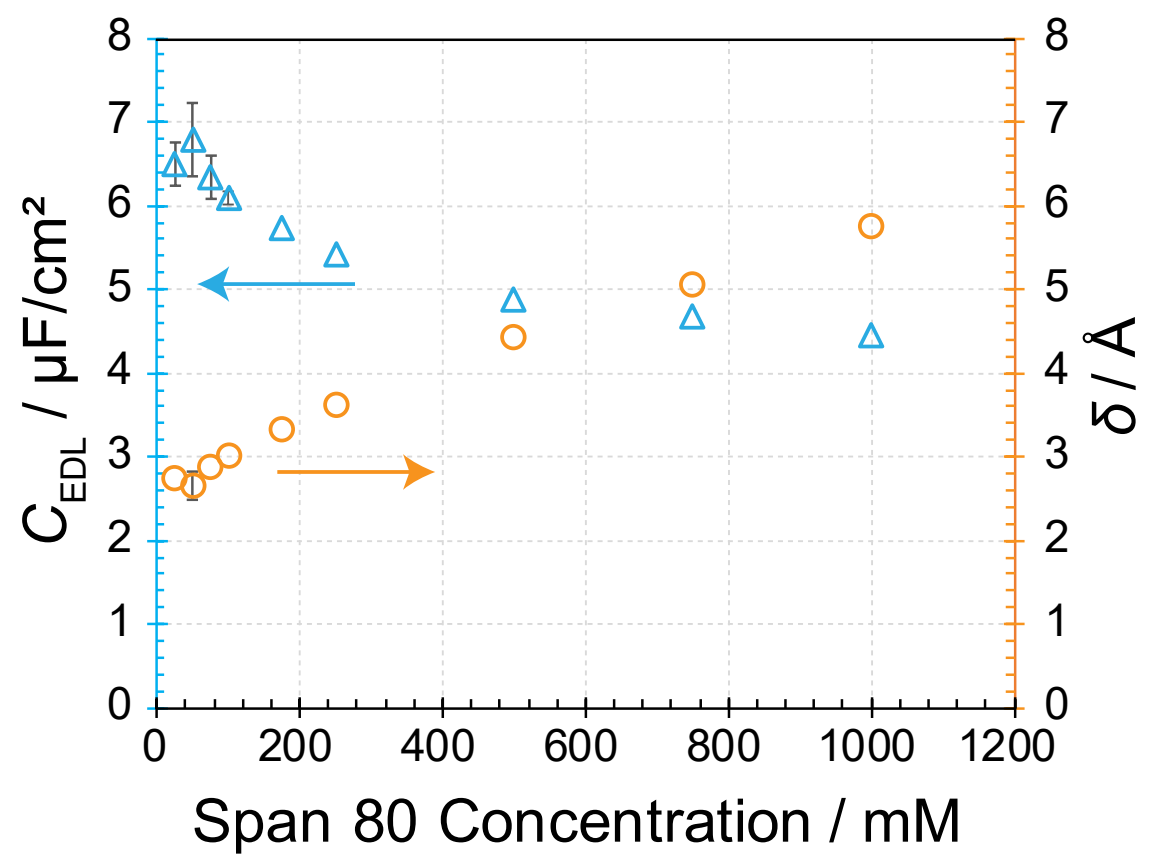

Figure 10: Specific EDL capacitance $C_{E D L}$ (triangles) and the electrode distance $\delta$ of an equivalent parallel-plate capacitor (circle) versus the concentration of Span 80.

field, we can assume the same permittivity value for the liquid bulk and the EDL. Therefore, the equivalent capacitance along with the respective permittivities and Equation (4) allows us to calculate the electrode distance $\delta$ of an equivalent parallel-plate capacitor. Given the parallel, planar and very smooth nature of our electrodes, this is a very reasonable approach. We plot this electrode distance as a function of surfactant concentration in Figure 10 (right axis) as open circles. Doing so, we find that this electrode distance slightly increases with increasing surfactant concentration but stays always on the order of magnitude of $1 \AA$.

According to the Gouy-Chapman-Stern model, the EDL consists of an immobile Stern layer and a mobile diffuse layer. Consequently, the overall EDL capacitance corresponds to a serial combination of the Stern layer capacitance and the diffuse layer capacitance. In our similar work on EIS in aqueous solutions, we showed that at very low ionic strengths, the overall EDL capacitance is dominated by the Stern layer capacitance. ${ }^{68}$ This is not in agreement with the expectation of retrieving the lower capacitance. At low ionic strengths, 
the diffuse layer capacitor should be the dominating capacitance for the two capacitors in series. We related this controversial finding to the very low concentration of the ionic species. The results indicated that a certain ionic strength is required to form a measurable diffuse layer capacitor. Below that certain ionic strength, the diffuse layer probably does not form accordingly or does not behave like a (ideal) capacitor. In the present work, where we can easily adjust extremely low ionic strengths in the medium, we have also strong indications that we measure the Stern layer capacitance. This supports our concept of a minimum ionic strength for diffuse layer formation. The thickness calculated from Equation (4) and the EDL capacitance should therefore be considered as the thickness of an EDL which consists of a sole Stern layer. Consequently, the concept of the Debye length, which is based on the GouyChapman theory of a diffuse layer, should not be applied at very low ionic strengths. The decrease in the EDL capacitance and increase in apparent Stern layer thickness as a function of the concentration, shown in Figure 10, should be attributed to adsorption of neutral (surfactant) species which results in the displacement of the counter-ions (charged inverse micelles); cf. Ref. ${ }^{69}$ Dukhin et al. ${ }^{70}$ also investigated the influence of nonionic surfactant adsorption on the Stern layer (slipping plane) of alumina particles dispersed in an aqueous electrolyte. Despite the fundamental differences, there are still similarities to our work. The nonionic surfactant Tween 80 considerably reduced the alumina zeta potential by shifting the slipping plane but only in the range of moderate ionic strengths. There was essentially no influence of the surfactant on the zeta potential observed at high ionic strength, where the diffuse layer is suppressed which is comparable to our case of extreme low ionic strength where the diffuse layer is not formed. 


\section{Summary}

The focus of the present study is the investigation of the charge carrier and electrical double layer formation in a nonpolar medium in the presence of a nonionic surfactant. For this purpose, we employ electrical impedance spectroscopy to measure liquid bulk and EDL properties in two custom made cells filled with samples of Span 80 in dodecane in the concentration range of 1 to $1000 \mathrm{mM}$.

A typical spectrum measured in this study can be divided into three different regions: (i) a high frequency liquid bulk semicircle; (ii) a mid-range frequency transport region; and (iii) a low frequency EDL region. From the liquid bulk semicircle data, we extract the medium permittivity and conductivity. It is found that the medium permittivity is a linear function of the surfactant concentration. The medium conductivity increases with the surfactant concentration which indicates that the nonionic surfactant causes a charge carrier formation. Conductivity data are adjusted with the viscosity of the medium and a linear relationship is found for surfactant concentrations up to $250 \mathrm{mM}$. This linearity reveals that the charge formation obeys a disproportionation mechanism. We calculate that the average fraction of charged inverse micelles in the concentration range of $25 \mathrm{mM}$ to 250 $\mathrm{mM}$ corresponds to around $3.2 \cdot 10^{-5}$ while the Debye length varies from $250 \mathrm{~nm}$ to $80 \mathrm{~nm}$.

The equivalent capacitance of the electrical double layer is retrieved from the low frequency EDL data of the spectra. For the entire range of concentration, the equivalent capacitance varies only slightly from around 7 to $4 \mu \mathrm{F} / \mathrm{cm}^{2}$. Keeping the parallel, planar and smooth nature of our electrodes in my mind, the electrode distance of an equivalent parallel plate capacitor is found to be on the order of angstrom. In other words, the Stern layer capacitance is measured in our EIS measurements and no significance of a diffuse layer

in determining the equivalent capacitance is found in this study. Hence, the concept of the Debye length should be avoided in media with very low ionic strength. 


\section{Acknowledgement}

The authors thank E.I. DuPont Canada Co. and NSERC for financial support.

\section{References}

(1) Lyklema, J. Principles of interactions in non-aqueous electrolyte solutions. Current Opinion in Colloid and Interface Science 2013, 18, 116-128.

(2) Smith, G. N.; Eastoe, J. Controlling colloid charge in nonpolar liquids with surfactants. Physical Chemistry Chemical Physics 2013, 15, 424-439.

(3) Prieve, D. C.; Yezer, B. A.; Khair, A. S.; Sides, P. J.; Schneider, J. W. Formation of charge carriers in liquids. Advances in Colloid and Interface Science 2017, 244, 21-35.

(4) Morrison, I. D. Electrical charges in nonaqueous media. Colloids and Surfaces A: Physicochemical and Engineering Aspects 1993, 71, 1-37.

(5) Beunis, F.; Strubbe, F.; Karvar, M.; Drobchak, O.; Brans, T.; Neyts, K. Inverse micelles as charge carriers in nonpolar liquids: Characterization with current measurements. Current Opinion in Colloid and Interface Science 2013, 18, 129-136.

(6) Dukhin, A.; Parlia, S. Ions, ion pairs and inverse micelles in non-polar media. Current Opinion in Colloid and Interface Science 2013, 18, 93-115.

(7) Gacek, M. M.; Berg, J. C. The role of acid-base effects on particle charging in apolar media. Advances in Colloid and Interface Science 2015, 220, 108-123.

(8) Suh, Y. K. Modeling and simulation of ion transport in dielectric liquids - Fundamentals and review. IEEE Transactions on Dielectrics and Electrical Insulation 2012, 19, 831848. 
(9) Strubbe, F.; Neyts, K. Charge transport by inverse micelles in non-polar media. Journal of Physics Condensed Matter 2017, 29, 453003.

(10) Barz, D. P. J.; Vogel, M. J.; Steen, P. H. Determination of the zeta potential of porous substrates by droplet deflection: II. Generation of electrokinetic flow in a nonpolar liquid. Langmuir 2010, 26, 3126-3133.

(11) Tolpekin, V. A.; Van Den Ende, D.; Duits, M. H.; Mellema, J. Flow electrification in nonaqueous colloidal suspensions, studied with video microscopy. Langmuir 2004, 20, $8460-8467$.

(12) Cabaleiro, J. M.; Paillat, T.; Moreau, O.; Touchard, G. Electrical double layer's development analysis: Application to flow electrification in power transformers. IEEE Transactions on Industry Applications 2009, 45, 597-605.

(13) Hao, T. Electrorheological fluids. Advanced Materials 2001, 13, 1847.

(14) Sheng, P.; Wen, W. Electrorheological fluids: Mechanisms, dynamics, and microfluidics applications. Annual Review of Fluid Mechanics 2012, 44, 143-174.

(15) Comiskey, B.; Albert, J. D.; Yoshizawa, H.; Jacobson, J. An electrophoretic ink for all-printed reflective electronic displays. Nature 1998, 394, 253-255.

(16) Bert, T.; Beunis, F.; Smet, H. D.; Neyts, K. Steady state current in EPIDs. Displays 2006, 27, 35-38.

(17) Heikenfeld, J.; Drzaic, P.; Yeo, J.-S.; Koch, T. Review Paper: A critical review of the present and future prospects for electronic paper. Journal of the Society for Information Display 2011, 19, 129.

(18) Chen, I.; Mort, J.; Machonkin, M. A. Particle charge, mobility, and $\zeta$ Potential in nonpolar colloids; 1997; Vol. 13; pp 5036-5039. 
(19) Jones, S. A.; Martin, G. P.; Brown, M. B. Manipulation of beclomethasonehydrofluoroalkane interactions using biocompatible macromolecules. Journal of Pharmaceutical Sciences 2006, 95, 1060-1074.

(20) Rosenholm, J. B. Solvent and surfactant induced interactions in drug dispersions. Colloids and Surfaces A: Physicochemical and Engineering Aspects 2010, 354, 197-204.

(21) Briscoe, W. H.; Horn, R. G. Direct measurement of surface forces due to charging of solids immersed in a nonpolar liquid. Langmuir 2002, 18, 3945-3956.

(22) Hsu, M. F.; Dufresne, E. R.; Weitz, D. A. Charge stabilization in nonpolar solvents. Langmuir 2005, 21, 4881-4887.

(23) Kim, J.; Anderson, J. L.; Garoff, S.; Schlangen, L. J. Ionic conduction and electrode polarization in a doped nonpolar liquid. Langmuir 2005, 21, 8620-8629.

(24) Dukhin, A. S.; Goetz, P. J. How non-ionic "electrically neutral" surfactants enhance electrical conductivity and ion stability in non-polar liquids. Journal of Electroanalytical Chemistry 2006, 588, 44-50.

(25) Roberts, G. S.; Sanchez, R.; Kemp, R.; Wood, T.; Bartlett, P. Electrostatic charging of nonpolar colloids by reverse micelles. Langmuir 2008, 24, 6530-6541.

(26) Kemp, R.; Sanchez, R.; Mutch, K. J.; Bartlett, P. Nanoparticle charge control in nonpolar liquids: Insights from small-angle neutron scattering and microelectrophoresis. Langmuir 2010, 26, 6967-6976.

(27) Guo, Q.; Singh, V.; Behrens, S. H. Electric charging in nonpolar liquids because of nonionizable surfactants. Langmuir 2010, 26, 3203-3207.

(28) Gacek, M. M.; Berg, J. C. Effect of surfactant hydrophile-lipophile balance (HLB) value on mineral oxide charging in apolar media. Journal of Colloid and Interface Science 2015, 449, 192-197. 
(29) Griffin Smith, P.; Patel, M. N.; Kim, J.; Milner, T. E.; Johnston, K. P. Effect of surface hydrophilicity on charging mechanism of colloids in low-permittivity solvents. Journal of Physical Chemistry C 2007, 111, 840-848.

(30) Espinosa, C. E.; Guo, Q.; Singh, V.; Behrens, S. H. Particle charging and charge screening in nonpolar dispersions with nonionic surfactants. Langmuir 2010, 26, 1694116948.

(31) Poovarodom, S.; Berg, J. C. Effect of particle and surfactant acid-base properties on charging of colloids in apolar media. Journal of Colloid and Interface Science 2010, $346,370-377$.

(32) Gacek, M. M.; Berg, J. C. Investigation of surfactant mediated acid-base charging of mineral oxide particles dispersed in apolar systems. Langmuir 2012, 28, 17841-17845.

(33) Smith, G. N.; Ahualli, S.; Delgado, Á. V.; Gillespie, D. A.; Kemp, R.; Peach, J.; Pegg, J. C.; Rogers, S. E.; Shebanova, O.; Smith, N.; Eastoe, J. Charging poly(methyl methacrylate) latexes in nonpolar solvents: Effect of particle concentration. Langmuir 2017, 33, 13543-13553.

(34) Strubbe, F.; Verschueren, A. R.; Schlangen, L. J.; Beunis, F.; Neyts, K. Generation current of charged micelles in nonaqueous liquids: Measurements and simulations. Journal of Colloid and Interface Science 2006, 300, 396-403.

(35) Karvar, M.; Strubbe, F.; Beunis, F.; Kemp, R.; Smith, A.; Goulding, M.; Neyts, K. Transport of charged aerosol OT inverse micelles in nonpolar liquids. Langmuir $\mathbf{2 0 1 1}$, 27, $10386-10391$.

(36) Parent, M. E.; Yang, J.; Jeon, Y.; Toney, M. F.; Zhou, Z. L.; Henze, D. Influence of surfactant structure on reverse micelle size and charge for nonpolar electrophoretic inks. Langmuir 2011, 27, 11845-11851. 
(37) Karvar, M.; Strubbe, F.; Beunis, F.; Kemp, R.; Smith, N.; Goulding, M.; Neyts, K. Investigation of various types of inverse micelles in nonpolar liquids using transient current measurements. Langmuir 2014, 30, 12138-12143.

(38) Karvar, M.; Strubbe, F.; Beunis, F.; Kemp, R.; Smith, N.; Goulding, M.; Neyts, K. Charging dynamics of Aerosol OT inverse micelles. Langmuir 2015, 31, 10939-10945.

(39) Strubbe, F.; Prasad, M.; Beunis, F. Characterizing generated charged inverse micelles with transient current measurements. Journal of Physical Chemistry A 2015, 119, $1957-1965$.

(40) Prasad, M.; Beunis, F.; Neyts, K.; Strubbe, F. Switching of charged inverse micelles in non-polar liquids. Journal of Colloid and Interface Science 2015, 458, 39-44.

(41) Prasad, M.; Strubbe, F.; Beunis, F.; Neyts, K. Different types of charged-inverse micelles in nonpolar media. Langmuir 2016, 32, 5796-5801.

(42) Prieve, D. C.; Hoggard, J. D.; Fu, R.; Sides, P. J.; Bethea, R. Two independent measurements of Debye lengths in doped nonpolar liquids. Langmuir 2008, 24, 1120-1132.

(43) Yezer, B. A.; Khair, A. S.; Sides, P. J.; Prieve, D. C. Use of electrochemical impedance spectroscopy to determine double-layer capacitance in doped nonpolar liquids. Journal of Colloid and Interface Science 2015, 449, 2-12.

(44) Yezer, B. A.; Khair, A. S.; Sides, P. J.; Prieve, D. C. Determination of charge carrier concentration in doped nonpolar liquids by impedance spectroscopy in the presence of charge adsorption. Journal of Colloid and Interface Science 2016, 469, 325-337.

(45) Peltonen, L.; Hirvonen, J.; Yliruusi, J. The behavior of sorbitan surfactants at the water-oil interface: Straight-chained hydrocarbons from pentane to dodecane as an oil phase. Journal of Colloid and Interface Science 2001, 240, 272-276. 
(46) Dukhin, A.; Parlia, S.; Somasundaran, P. Rheology of non-Newtonian liquid mixtures and the role of molecular chain length. Journal of Colloid and Interface Science $\mathbf{2 0 2 0}$, $560,492-501$.

(47) Abou-Nemeh, I.; Bart, H. J. Microstructures in the system water/D2EHPA/Span-80/ $n$-dodecane; 1998; Vol. 14; pp 4451-4459.

(48) Dukhin, A.; Parlia, S.; Somasundaran, P. Ion-Pair Conductivity Theory V: Critical Ion Size and Range of Ion-Pair Existence. Journal of The Electrochemical Society 2018, 165, E784-E792.

(49) Kopanichuk, I. V.; Vedenchuk, E. A.; Koneva, A. S.; Vanin, A. A. Structural properties of span 80 /tween 80 reverse micelles by molecular dynamics simulations. Journal of Physical Chemistry B 2018, 122, 8047-8055.

(50) MacDonald, J. Comparison and discussion of some theories of the equilibrium electrical double layer in liquid electrolytes. Journal of Electroanalytical Chemistry and Interfacial Electrochemistry 1987, 223, 1-23.

(51) MacDonald, J. R. Impedance spectroscopy. Annals of Biomedical Engineering 1992, 20, 289-305.

(52) Barbero, G. Theoretical interpretation of Warburg's impedance in unsupported electrolytic cells. Physical Chemistry Chemical Physics 2017, 19, 32575-32579.

(53) Barbero, G.; Lelidis, I. Analysis of Warburg's impedance and its equivalent electric circuits. Physical Chemistry Chemical Physics 2017, 19, 24934-24944.

(54) Zoltowski, P. On the electrical capacitance of interfaces exhibiting constant phase element behaviour. Journal of Electroanalytical Chemistry 1998, 443, 149-154.

(55) Sadkowski, A. On the ideal polarisability of electrodes displaying CPE-type capacitance dispersion. Journal of Electroanalytical Chemistry 2000, 481, 222-226. 
(56) Hirschorn, B.; Orazem, M. E.; Tribollet, B.; Vivier, V.; Frateur, I.; Musiani, M. Determination of effective capacitance and film thickness from constant-phase-element parameters. Electrochimica Acta 2010, 55, 6218-6227.

(57) Schmidt, J.; Prignitz, R.; Peschka, D.; Münch, A.; Wagner, B.; Bänsch, E.; Peukert, W. Conductivity in nonpolar media: Experimental and numerical studies on sodium AOT-hexadecane, lecithin-hexadecane and aluminum(III)-3,5-diisopropyl salicylatehexadecane systems. Journal of Colloid and Interface Science 2012, 386, 240-251.

(58) Gourdin-Bertin, S.; Chassagne, C. Application of classical thermodynamics to the conductivity in non-polar media. Journal of Chemical Physics 2016, 144, 244501.

(59) Parlia, S.; Dukhin, A.; Somasundaran, P. Ion-pair conductivity theory: Mixtures of non-polar media with non-ionic surfactant. Journal of the Electrochemical Society 2017, 164, E295-E299.

(60) Parlia, S.; Somasundaran, P.; Dukhin, A. Ion-pair conductivity theory IV: SPAN surfactants in toluene and the role of viscosity. Journal of the Electrochemical Society 2018, 165, H170-H175.

(61) Dukhin, A. Critical concentration of ion-pairs formation in nonpolar media. Electrophoresis 2014, 35, 1773-1781.

(62) Dukhin, A.; Parlia, S. Ion-pair conductivity theory fitting measured data for various alcohol-toluene mixtures across entire concentration range. Journal of the Electrochemical Society 2015, 162, H256-H263.

(63) Parlia, S.; Dukhin, A.; Somasundaran, P. Ion-pair conductivity theory: Mixtures of butanol with various non-polar liquids and water. Journal of The Electrochemical Society 2016, 163, H570-H575. 
(64) Beunis, F.; Strubbe, F.; Marescaux, M.; Neyts, K.; Verschueren, A. R. Diffuse double layer charging in nonpolar liquids. Applied Physics Letters 2007, 91.

(65) Smith, G. N.; Brown, P.; James, C.; Kemp, R.; Khan, A. M.; Plivelic, T. S.; Rogers, S. E.; Eastoe, J. The effects of counterion exchange on charge stabilization for anionic surfactants in nonpolar solvents. Journal of Colloid and Interface Science 2016, 465, 316-322.

(66) Brug, G. J.; van den Eeden, A. L.; Sluyters-Rehbach, M.; Sluyters, J. H. The analysis of electrode impedances complicated by the presence of a constant phase element. Journal of Electroanalytical Chemistry 1984, 176, 275-295.

(67) Orazem, M. E.; Frateur, I.; Tribollet, B.; Vivier, V.; Marcelin, S.; Pebere, N.; Bunge, A. L.; White, E. A.; Riemer, D. P.; Musiani, M. Dielectric properties of materials showing constant-phase-element (CPE) impedance response. Journal of the Electrochemical Society 2013, 160, C215-C225.

(68) Khademi, M.; Barz, D. P. J. Electrical Double Layer Structure Revisited: Electrode Capacitance in Aqueous Electrolyte and Surfactant Solutions. Langmuir 2020, accepted.

(69) Vincent, B.; Bijsterbosch, B.; Lyklema, J. Competitive adsorption of ions and neutral molecules in the stern layer on silver iodide and its effect on colloid stability. Journal of Colloid and Interface Science 1971, 3\%, 171-178.

(70) Dukhin, A.; Dukhin, S.; Goetz, P. Electrokinetics at high ionic strength and hypothesis of the double layer with zero surface charge. Langmuir 2005, 21, 9990-9997. 
TOC Graphic

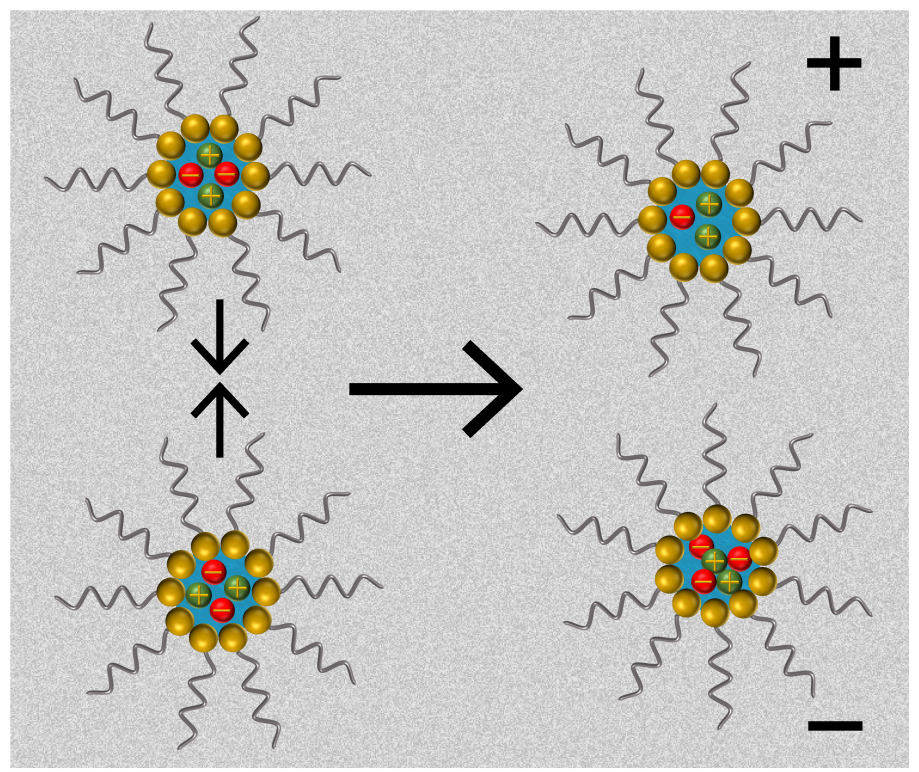

